\title{
Olfaction and Identification of Unrelated Individuals: Examination of the Mysteries of Human Odor Recognition
}

\author{
Shannon B. Olsson • Joan Barnard • Linda Turri
}

Received: 14 September 2005 / Revised: 23 November 2005 /

Accepted: 28 November 2005 / Published online: 2 August 2006

(C) Springer Science + Business Media, Inc. 2006

\begin{abstract}
Although several studies have examined the effect of human odor on kin recognition and mate choice, few have focused on the impact of familiarity on recognition of nonrelatives by olfactory cues. As part of a program designed to engage students in scientific research, 53 high school students researched, planned, and implemented a project to analyze the effect of odor on human recognition of, and preference for, friends, sex, and self. A total of 37 students, including friends of their choosing, wore T-shirts for three consecutive nights. During that time, subjects were controlled for exposure to extraneous perfumes, household odors, and other humans. The students were then asked to smell a series of five shirts and evaluate them with respect to pleasantness. Students were also asked to identify the shirts belonging to themselves and their friend, and determine the sex of the person who wore each shirt. Although subjects were unable to distinguish sex by olfactory cues alone, a significant percentage of subjects were able to identify their own odor $(51.6 \%)$, as well as distinguish the odor cue of their friend (38.7\%). Additionally, subjects who could not identify their friend's cue were apt to choose the odor of a member of the opposite sex as their friend. This result was not believed to rely on odor preference as neither individual, friend, nor stranger odors were rated significantly different with respect to odor pleasantness. The ability to recognize friends via odor cues lends credence to the hypothesis that association and familiarity are important aspects of conspecific olfactory recognition in humans. Furthermore, this study augments evidence that olfaction may supplement visual and auditory cues used in human conspecific and kin recognition.
\end{abstract}

Keywords Kin recognition · Human social behavior - Association · Familiarity

This research was designed and implemented by 53 high school General Biology and Advanced Placement students who contributed as fellow authors to this project. Refer to Acknowledgments for a full list of names.

\footnotetext{
S. B. Olsson $(\bowtie)$

Department of Entomology, NYS Agricultural Experiment Station,

Cornell University, Geneva, NY 14456, USA

e-mail: sbb23@cornell.edu

J. Barnard · L. Turri

Biology Department, Geneva High School, Geneva, NY 14456, USA
} 


\section{Introduction}

"Reason is sight. Instinct is touch. Intuition is smell." Mason Cooley (2001)

Humans are creatures of sight and sound. Yet, of all the human senses, olfaction is considered the most ancient, possessing links to several areas of the brain, including those responsible for cognitive, visceral, emotional, and homeostatic behaviors (Purves et al., 2001). Moreover, although it is often assumed that humans have a poor sense of smell, we possess an amazingly acute sensitivity to some chemicals, such as vanillin and 2-bromophenol (sensitivity thresholds of $10^{-5.6}$ and $10^{-4.5} \mathrm{ppm}$, respectively; Devos et al., 1990; Jacob et al., 2002). In fact, humans can recognize at least 10,000 different odors (Prasad and Reed, 1999).

Humans may also use olfactory cues for conspecific recognition and communication (see Hays, 2003; Wysocki and Preti, 2000, 2004 for recent reviews). With its persistence and complexity, the olfactory cue is suitable for producing highly individualized and longlasting signals. Even with voices and faces, olfaction may function throughout life to supplement these cues. Furthermore, olfactory cues learned in the first few hours of life, or even prenatally, might impact our earliest lessons of whom kin and non-kin are (refer to Porter, 1999).

Odor-mediated recognition of conspecifics is ubiquitous throughout the animal kingdom. However, the mechanisms for discrimination can be diverse. For example, Polistes metricus paper wasps use the scent of hydrocarbons from their nest to recognize kin (Espelie et al., 1990). Salmonid fishes, well known for their use of olfaction in kin recognition, potentially use odors mediated by major histocompatibility complex (MHC) alleles (immunoresponse genes) to determine kin (Olsen et al., 1998; Rajakaruna et al., 2001). However, another fish, the Trinidadian guppy (Poecilia reticulata), uses odor to identify and school with familiar conspecifics, whether they are genetically related or not (Griffiths and Magurran, 1999).

In mammals, there are several examples of conspecific recognition via olfaction. Golden hamsters (Mesocricetus auratus) appear to use self-referent phenotype matching of odors (the "armpit effect") to determine kin (Mateo and Johnston, 2000a). Belding's ground squirrels (Spermophilus beldingi) respond toward littermate odors even after hibernation (Mateo and Johnston, 2000b). These squirrels can also discriminate degrees of relatedness through odor alone (Mateo, 2002). In this species, precise recognition may be mediated by both kin association and self or kin phenotype matching (Mateo and Johnston, 2000b).

There is a growing body of evidence that humans can also recognize each other via olfactory cues. Several studies have shown evidence for odor-mediated self-recognition (Russell, 1976; Hold and Schleidt, 1977; Schleidt, 1980; Schleidt et al., 1981; Lord and Kasprzak, 1989; Platek et al., 2001). Mothers can recognize olfactory cues from their infants within a few hours of birth (Porter and Cernoch, 1983; Russell et al., 1983; Kaitz et al., 1987). Likewise, infants will preferentially orient their heads toward maternal odors (Cernoch and Porter, 1985; Porter et al., 1992). There is also evidence that siblings and other relatives can recognize each other using odor cues (Porter and Moore, 1981; Porter et al., 1986).

The mechanisms underlying human odor recognition have not been elucidated. A number of studies have suggested that odor is influenced by the MHC, a series of genes also involved in immune response (see Penn, 2002 for review). In addition, both phenotype matching and association have been suggested to play a role in human kin recognition (Weisfeld et al., 2003). However, few studies have examined odor-mediated recognition of nonrelatives. Studies of relatives show that we can recognize conspecifics, but are unable to 
distinguish the mechanisms behind this recognition. Genetically related individuals might employ both association and phenotype matching to identify each other. Tests of nonrelatives are imperative to understand the impact of familiarity on human odor recognition, as phenotype matching is impossible where no genetic relatedness exists. Wallace (1977) found that subjects could distinguish between the hand odor of two different individuals. Kaitz and Eidelman (1992) showed that nonparturient strangers with little infant experience could distinguish a newborn's undershirt from other infants' after holding them for only $45 \mathrm{~min}$. Verron and Gaultier (1976) found that 4- to 5-yr-old children were able to identify peers to some extent, although it was unclear how they controlled for extraneous scents due to foods, perfumes, and other sources. In 1998, Mallet and Schaal also found that young school children (average age of $9.5 \mathrm{yr}$ ) were able to identify nonrelative peers. However, the authors were not interested in purely biological odors and did not control for any extraneous scents. The majority of other studies that have tackled human odor recognition have concentrated their efforts on the recognition of partners (Hold and Schleidt, 1977; Schleidt, 1980; Schleidt et al., 1981). These studies are insufficient to examine effects of familiarity because subjects were also influenced by sexual attraction. Weisfeld et al. (2003) might have examined nonrelatives other than partners; however, because they did not separate the two in their presentation, no conclusions can be drawn.

In the present study, we investigated the impact of familiarity and association on human odor recognition by testing the hypothesis that humans can recognize familiar nonrelatives by odor cues. As part of a program designed to engage students in scientific research, 53 General and Advanced Placement biology students (ages 15-18 yr) implemented a project to analyze the effect of odor on human recognition of, and preference for, friends, sex, and self. Thirty-seven students wore T-shirts for three consecutive nights. During that time, subjects were controlled for exposure to an extensive list of extraneous odors.

\section{Methods and Materials}

The study was approved by the Cornell University Committee on Human Subjects (Institutional Review Board) and the Geneva Central School District Administration for use of data collected for research purposes.

\section{Subjects}

A total of 37 Geneva High School students (Geneva, NY, USA; 26 females, 11 males), ages 15-18 yr $(\bar{X}=16.77)$, volunteered to participate in a study to examine human odor recognition of, and preference for friends, sex, and self. This population was diverse on both racial and socioeconomic levels. Students were asked to choose one friend to participate in the experiment with them. They were also asked to choose only friends with whom they had a platonic relationship. Of the pairings used for data analysis, 2 were male/ male, 12 were female/female, and 7 were male/female.

Thirty-one students were members of classes participating in a university and National Science Foundation-sponsored program designed to engage students in scientific research. The two class instructors participated in the program and coordinated with S. Olsson to integrate inquiry research into their General and Advanced Placement Biology curriculum. As such, the entire class designed and implemented all aspects of this project as part of the curriculum and was informed of the testing procedures. The six students from other classes 
were informed both by written and oral instructions from the student friend participating in the program. All class members were educated on the ethics of human subject research both for research training and to inform them of their rights as potential subjects. All information and case studies for the education were taken directly from The Cornell University Committee on Human Subjects Training Program for Researchers in the Use of Human Subjects, and The National Institutes of Health Human Participant Protections Education for Research Terms on-line tutorial. Students were assessed through in-class discussions of case studies for their understanding of autonomy, beneficence, and justice as regards the use of human subjects in research. Although participation in planning and data analysis was a mandatory aspect of the education, participation as experimental subjects was strictly voluntary. Students were informed that any assessment for grading purposes was based solely on participation in planning and analysis of the study. Furthermore, students who did not volunteer as subjects were identical to subjects in their participation in all other aspects of the project. Thus, only 31 of the 52 total class members and six friends from other classes chose to participate. All student information was kept confidential through the use of identification numbers, and students were presented only these numbers during the data analysis phase. Consequently, data records could not be traced to specific students.

\section{Odor Collection and Storage}

Subjects were provided with $100 \%$ cotton T-shirts to wear to bed on three consecutive nights with clean pajama or alternative bottoms. Before wearing the shirts, subjects were instructed to wash all bed sheets in an unscented laundry detergent provided to them. The 37 shirts as well as one unworn T-shirt used as a control were laundered together in the same detergent before wearing. Subjects were also presented with the same lightly scented shampoo, conditioner, and soap (Zoto International, Inc.) to use each night immediately before donning the shirt.

Subjects were instructed to use only the items provided to them, and avoid other soaps, lotions, deodorants, etc. Although they were not required to alter daytime activities, subjects were cautioned to avoid smoking, eating, and strenuous activity while wearing the shirts. Finally, they were instructed to wear the shirts at least $6 \mathrm{hr}$ each night, and prevent other humans/pets from sleeping on or using the bed during the testing period. Each morning, subjects placed their shirts in a plastic zip-lock bag to store in the freezer during the day to minimize loss of odor. Upon collection, all sealed bags, including the unworn control in a sealed bag, were stored together in the same freezer at approximately $-5^{\circ} \mathrm{C}$.

\section{Testing Procedure}

Subjects were tested by S. Olsson on 3 consecutive days in January 2004, approximately 6$8 \mathrm{~d}$ after wearing the shirts. They were first asked to complete a questionnaire concerning the study, providing data such as age, sex, information about their friendships (length, number of days a week they met their friend, etc.), compliance with testing procedures, information about potential impediments to the study such as allergies or illness that could create olfactory deficits, and exposure to pets smoke and strong food odors that could facilitate identification of specific individuals.

Upon completion of the questionnaire, subjects were screened for olfactory deficits by identifying a sequence of three scents [orange, vanilla, and almond extracts (McCormick and Co., Inc., Hunt Valley, MD, USA)] diluted 50\% in water, similar to screening methods described in another human odor recognition study (Kaitz et al., 1987). Subjects were asked 
to identify the three correct scents from a list of 10 odor choices. Then, wearing latex or nitrile gloves, they were asked to open and smell the contents of five numbered zip-lock bags containing shirts worn by themselves, their friend, an unworn control, and two strangers from the opposite sex. The unworn control had been laundered and stored at $-5^{\circ} \mathrm{C}$ with the worn T-shirts. The two "strangers" were subjects randomly chosen from classes other than the test subject. Strangers of the opposite sex were used to aid in testing odor recognition of sex. Bags and shirts were of similar size and color with no identifying marks. The bags were numbered 1-5 and presented in random order. Subjects were allowed to smell the contents as long as necessary and repeat if needed to complete a test.

In the first test, subjects rated the pleasantness of each shirt's odor on a scale from 1-5 (1, very unpleasant; 2, somewhat unpleasant; 3, neutral; 4, somewhat pleasant; 5, very pleasant). They were then asked to determine the sex of each of the five shirt owners (including the unworn control shirt). Finally, each was asked to identify the two shirts belonging to self and friend.

\section{Statistical Analyses}

$\chi^{2}$ Tests (StatXact-4 and StatXact-6, Cytel Software Corporation, 1998 and 2004) were used to compare pleasantness ratings for each type of shirt (Pearson's with Monte Carlo estimates of exact $P$ values using 10,000 samples) as well as compare frequencies of shirts chosen as self and friend to the $20 \%$ expected by chance (goodness-of-fit with exact $P$ values). All $\chi^{2} P$ values reported are exact. A Kolmogorov-Smirnov $Z$ test was used to compare distributions of pleasantness ratings for the tested shirt types (SPSS Version 12.0, SPSS, Inc., 2001). For recognition of sex, a logistic model was fitted to the number of correct and incorrect selections for each individual using STATA (v.7, StataCorp, 2001) with a Huber-White robust variance estimator to obtain $95 \%$ confidence intervals, which accounted for positive correlation with individual. Finally, logistic regression (STATA v. 7, StataCorp, 2001) was used to determine the influence of a number of factors answered in the questionnaire (see testing procedure, above) on identification of self and friend.

\section{Results}

\section{Olfactory Screening}

Subjects were initially screened for olfactory deficits through identification of three odors: orange, vanilla, and almond. Only those who correctly identified at least two of three odorants were considered for further analysis. However, 20/37 subjects (54.0\%) identified orange odor as lemon. Because of this, lemon was accepted as an alternate response to orange. Most of the subjects $(n=31,83.8 \%)$ identified at least two of three odorants correctly, including those who chose lemon.

\section{Human Odor Qualitative Comparisons}

Each subject rated the pleasantness of five T-shirts (self, friend, male stranger, female stranger, and an unworn, blank shirt) on a scale of 1-5. Figure 1 depicts the distribution of pleasantness ratings for each of the five types of shirts. All distributions are similar with the exception of the unworn, blank shirt, which was considered neutral more frequently. According to a $\chi^{2}$ test, the distribution of pleasantness ratings did not differ among the shirt 


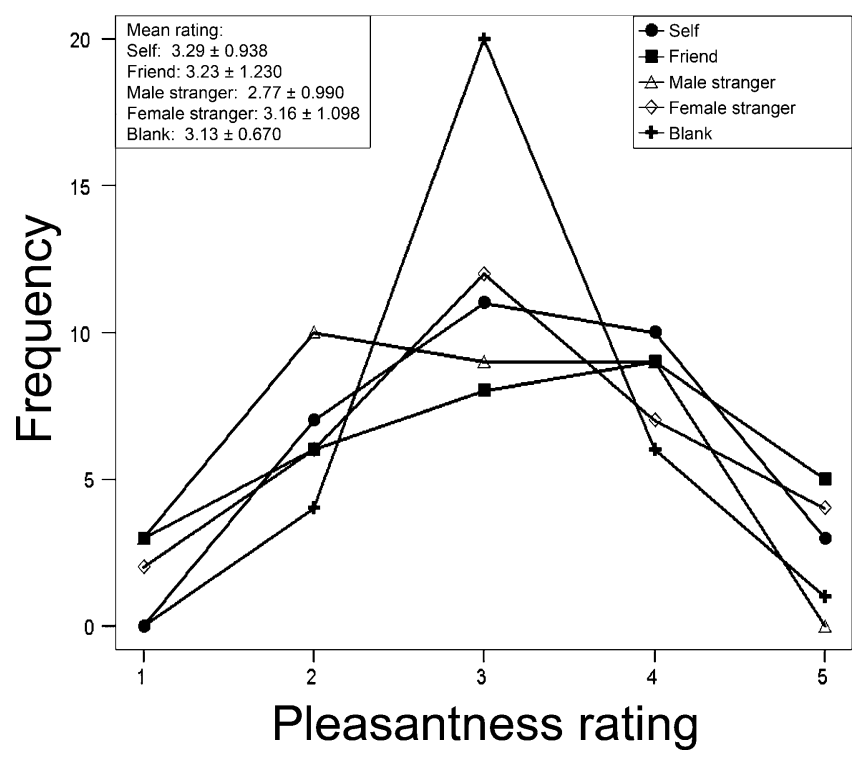

Fig. 1 Distributions of pleasantness for each of the five shirt types. Each shirt was rated 1-5, as follows: 1, very unpleasant; 2 , somewhat unpleasant; 3 , neutral; 4 , somewhat pleasant; 5 , very pleasant

types, $(d f=16, n=155)=24.09, P=0.08$, nonsignificant (NS). These distributions were also similar when male and female subjects were analyzed separately [data not shown; males: $\chi^{2}(d f=16, n=40)=22.81, P=0.110$, NS; females: $\chi^{2}(d f=16, n=115)=23.28$, $P=0.109$, NS. A Kolmogorov-Smirnov $Z$ test also did not find any difference between any paired rating distributions $(Z=0.254-1.143, P>0.1)$.

\section{Recognition of Sex via Odor Cues}

Subjects were asked to identify the sex of the owner for each of the five shirts presented. A logistic model was fitted to these data with a robust variance estimator accounting for
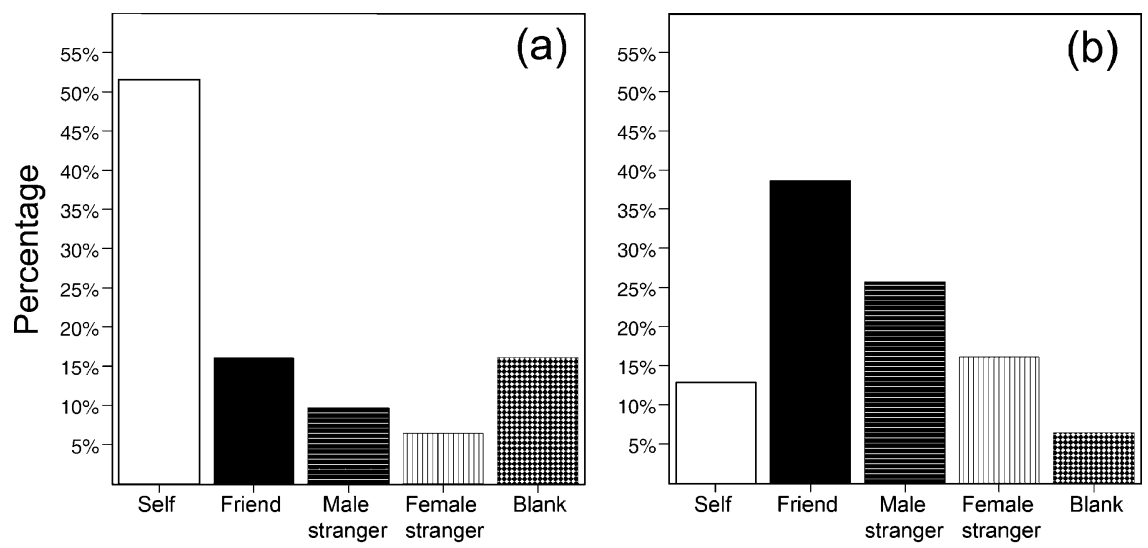

Shirt chosen

Fig. 2 Percentage of T-shirts chosen by the subjects as their (a) own shirt and (b) friend's shirt $(n=31)$ 
positive correlation with individual. On average, subjects identified $54.8 \pm 8 \%$ shirts correctly $(95 \%$ confidence interval), which does not differ significantly from chance. The unworn, blank shirt was identified male as frequently as it was identified female $(15 / 31$ or $48.4 \%$, and $16 / 31$ or $51.6 \%$, respectively). However, males were 1.67 times more likely to identify the blank shirt as male $(5: 3)$, whereas females were 1.30 times more likely to identify it as female (13:10).

\section{Self-Recognition via Odor Cues}

Figure 2a shows the distribution of shirts identified by the subjects as their own. A total of $51.6 \%$ of the subjects identified their own shirt correctly, which is significantly greater than the $20 \%$ expected by chance, $\chi^{2}(1, n=31)=19.36, P<0.001$. Females identified their own shirt significantly greater than chance, $\chi^{2}(1, n=23)=14.88, P<0.001$, and males were marginally significant (MS), $\chi^{2}(1, n=8)=4.5, P=0.056$, MS. During testing, four shirts were identified by at least two to three subjects as having a "nonhuman" odor, such as food or cigarette smoke. Removal of these shirts from all aspects of the data pool (self, friend, and stranger) did not effect this significance, $\chi^{2}(1, n=14)=12.07, P=0.002$. Likewise, Table 1 shows that subjects could still identify their own shirt even when controlling for several factors that could have impacted result accuracy. This table was compiled from subjects' questionnaire answers filled out the day of testing (see Methods and Materials). Subjects who answered "yes" to any factor such as allergies, sickness, or

Table 1 Recognition of self and friend odor cues as a function of specific conditions or situations

\begin{tabular}{|c|c|c|c|c|c|c|}
\hline \multirow[t]{2}{*}{ Condition controlled } & \multirow{2}{*}{$\begin{array}{l}\text { No. subjects who } \\
\text { answered "no" on } \\
\text { questionnaire }\end{array}$} & \multicolumn{2}{|c|}{ Self-recognition } & \multirow{2}{*}{$\begin{array}{l}\text { No. subjects whose } \\
\text { friends answered "no" } \\
\text { on questionnaire }\end{array}$} & \multicolumn{2}{|c|}{ Friend recognition } \\
\hline & & $\chi^{2}$ & $P$ value & & $\chi^{2}$ & $P$ value \\
\hline Pet owners & 9 & 12.25 & 0.003 & 8 & 0.12 & $1.000, \mathrm{NS}$ \\
\hline Smokers ${ }^{\mathrm{a}}$ & 24 & 10.01 & 0.004 & 25 & 4.00 & $0.074, \mathrm{NS}$ \\
\hline Allergies & 23 & 14.88 & $<0.001$ & $23^{\mathrm{b}}$ & 7.92 & $0.009^{\mathrm{b}}$ \\
\hline $\begin{array}{l}\text { Strong foods } \\
\text { consumed } \\
\text { during testing }\end{array}$ & 23 & 24.01 & $<0.001$ & 24 & 7.04 & 0.013 \\
\hline $\begin{array}{l}\text { Odorants used (i.e., } \\
\text { perfumes, lotions, } \\
\text { deodorant) }\end{array}$ & 28 & 19.72 & $<0.001$ & 27 & 4.90 & 0.033 \\
\hline $\begin{array}{l}\text { Illness while } \\
\text { wearing } \\
\text { shirt or during } \\
\text { olfactory trials }\end{array}$ & 22 & 16.41 & $<0.001$ & $22^{\mathrm{c}}$ & 3.68 & $0.063, \mathrm{MS}^{\mathrm{c}}$ \\
\hline $\begin{array}{l}\text { Directions not } \\
\text { followed (e.g., } \\
\text { proper soaps not } \\
\text { used, shirt not } \\
\text { worn three } \\
\text { nights, etc.) }\end{array}$ & 20 & 15.31 & $<0.001$ & 23 & 5.26 & 0.033 \\
\hline
\end{tabular}

${ }^{a}$ Subjects or friends with household members who smoke are also excluded.

b Includes only subjects who answered "no" on questionnaire, but does not exclude any friends.

c Includes only subjects who answered "no" to illness during trials and whose friends answered "no" to illness while wearing shirt. 
exposure to smoke and strong food odors were removed from that analysis. A $\chi^{2}$ test was performed using only those subjects who stated they were not affected by that factor. The table lists the number of remaining subjects for each condition considered (i.e., those who answered "no" on questionnaire). Additionally, logistic regression found no influence of any of these factors on choice of self $(P>0.2)$.

\section{Recognition of Friends via Odor Cues}

Based on questionnaire answers, most of the subjects $(34 / 37,91.9 \%)$ considered their chosen partner to be a friend rather than simply a classmate. Nevertheless, only 19/37 (51.4\%) considered this person to be a close friend. All but one subject indicated that they met their friend at least three times a week, and 26/37 (70.3\%) stated that they had known their friend at least 3 yrs.

A total of $38.7 \%$ of the subjects identified their friend's shirt correctly, which is significantly greater than the $20 \%$ expected by chance, $\chi^{2}(1, n=31)=6.78, P=0.014$ (Fig. 2b). This was only true for friends who knew each other at least $3 \mathrm{yr}, \chi^{2}(1, n=21)=$ $6.86, P=0.014$. Friends who knew each other less than $3 \mathrm{yr}$ could not distinguish each other by odor, $\chi^{2}(1, n=10)=0.62, P=0.698$, NS. However, logistic regression found no influence of friendship length on subjects' ability to distinguish their friends' odor $(P>0.2)$.

Removal of the four shirts identified two to three times as having a "nonhuman" odor from the data pool did affect the significance, $\chi^{2}(1, n=14)=2.16, P=0.174$, NS. Nevertheless, Table 1 shows that subjects could still identify their friends even when their friends were controlled for most factors obtained from the questionnaire filled out the day of testing (i.e., only subjects whose friends stated they were not affected by that factor were included). The use of logistic regression found no influence of these factors on choice of friend $(P>0.2)$.

Finally, there was a sex difference in the ability of subjects to identify their friend's shirt. Although females could identify their friends, $\chi^{2}(1, n=23)=11.13, P=0.002$, males could not, $\chi^{2}(1, n=8)=0.28, P=0.706$, NS. However, the low sample size could have contributed to the lack of significance among male subjects. Additionally, if subjects selected incorrectly, males were four times more likely to identify the female stranger as their friend than the male stranger $(4: 1)$, whereas females were seven times more likely to identify the male stranger $(7: 1)$. The propensity to identify a member of the opposite sex as the friend was not dependent on the pairing of the subjects (male/male, female/female, and male/female). Indeed, in the 11 cases where female/female pairs identified the incorrect shirt, subjects selected the male stranger in seven of the cases (64\%). Likewise, in the three cases where male/male pairs identified friends incorrectly, they chose the female stranger once and their own shirt twice.

\section{Discussion}

Our results indicate that subjects, when presented with several different stimuli, could recognize both their own and their friend's odor cue. However, subjects could not reliably distinguish the sex of the wearer nor did they prefer the scent of any particular individual (i.e., self, friend, stranger, or unworn). Apparently, the ability to recognize odor in this context did not require a preference for that scent. All shirt odors were rated similarly for 
pleasantness, and although male stranger shirts were considered slightly less pleasant on average, this distinction was not significant.

Subjects were not only unable to distinguish sex via odor cues, but, as shown above, male and female odors were considered equally pleasant, indicating that neither sex possessed qualitative differences in odor. Furthermore, the unworn, blank control shirt was identified to be male as often as female, showing that subjects had no overall bias to choose one sex over the other. Nonetheless, both males from females were slightly more likely to choose their own sex for the unworn shirt. These results are in disagreement with previous studies (Hold and Schleidt, 1977; Schleidt, 1980; Schleidt et al., 1981). In these experiments, subjects (20-64\%) were not only able to discriminate males from females, but many deemed female odor to be "more pleasant" than male. However, shirts were worn for a longer period of time (seven nights) and fewer controls were taken (i.e., subjects' bed sheets not washed in same detergent, same shampoo not used by all subjects, shirts not stored under isolated, cold conditions, and ownership of pets not considered). Consequently, there was a greater potential for extraneous and unnatural odors to accumulate on the shirts and affect the results. Several studies reviewed in Doty (1981) also suggest the discrimination of sex via olfactory cues, and in these studies shirts/gauze pads were worn for 18-24 hr. In our study, subjects might have been able to distinguish sex had they worn the shirts for an extended period of time. It is also possible that the odor cues responsible for distinguishing men from women are less prominent during young adulthood than for adults used in the previous research. Lord and Kasprzak (1989) found that age was a significant factor in subjects' ability to recognize self via olfaction. In fact, many of the subjects who failed the test were under $20 \mathrm{yr}$, similar to our subjects. Mallet and Schaal (1998) also found that 9-yr-old children were unable to determine the sex of their peers by smell alone. Finally, a study of volatiles emitted from subjects ages $26-75 \mathrm{yr}$ found a positive correlation in the concentration of 2-nonenal with age, with detectable amounts only found in persons age $40 \mathrm{yr}$ and above (Haze et al. 2001). Perhaps our subjects neither produce sufficient quantities of, nor are adequately familiar with the odor cues responsible for distinguishing sex.

In both recognition tests, our subjects were able to distinguish their own and their friend's odor cue. Subjects could identify their own scent $51.6 \%$ of the time, and their friend's $38.7 \%$. The ability to recognize self via odor cues was not affected even when separately controlling for factors that might influence shirt odor, e.g., pets, smoking, allergies, strong foods eaten, odorants used, illness, and experimental error. The ability to recognize friends via odor cues was affected only when nonhuman-scented shirts, friends who were pet owners, or friends who were smokers were removed from the data set.

These results are in congruence with previous studies that confirm the ability of humans to recognize self via olfactory cues (Russell, 1976; Hold and Schleidt, 1977; Schleidt, 1980; Schleidt et al., 1981; Lord and Kasprzak, 1989; Mallet and Schaal, 1998; Platek et al., 2001). Furthermore, the percentage of subjects able to recognize their own scent $(51.6 \%)$ falls well within values obtained in other studies [25\% in Schleidt et al. (1981) to 75\% in Russell (1976) and Lord and Kasprzak (1989)].

Individuals were able to identify their friend's odor although only half (51.4\%) stated that their partner was a close friend. Furthermore, the result that only friends who knew each other longer than $3 \mathrm{yr}$ could identify each other may indicate that a longer period of association is necessary to learn olfactory cues. However, because of the small sample size in relation to this question ( $n=10$ for friends $<3 \mathrm{yr}$ ), and the lack of statistical relation to length of friendship, this conclusion must be made with caution. Additional studies with larger sample sizes may elucidate the validity of this claim. 
Subjects who did not identify their friend's odor correctly were apt to choose the odor of a member of the opposite sex. This was true even for same-sex pairings, showing that individuals who chose incorrectly were not merely searching for odors of the same sex as their friend. This agrees with the conclusion that our subjects could not distinguish sex. Although subjects did not express a conscious preference for the odor of the opposite sex, this result indicates that they may consider the odor of the opposite sex to be a "friendly" odor. Implications of this on the role of odor in human mate preference are beyond the scope of this investigation and are not discussed here.

The ability to recognize friends via odor cues lends credence to the idea that association and familiarity are important aspects of conspecific olfactory recognition in humans. Further tests are necessary to determine the extent to which humans utilize association vs. phenotype matching for kin and conspecific recognition. Potentially, a study with adopted children and unknown relatives could address this concern. Adopted children, although lacking a coefficient of genetic relatedness, are kin in the human social context. Recognition of adopted children could occur purely through association. However, a recent study found that although mothers and siblings could identify odor of their biological children and siblings, they were unable to identify the odor of their stepchildren or stepsiblings (Weisfeld et al., 2003). Alternatively, relatives whom the subject has never met could only be identified through phenotype matching. These tests are essential to understand the mechanisms by which we use odor as a means for identification.

Although this examination uses indirect cues (i.e., T-shirts), there remains significant support for the recognition of nonrelatives via odor cues. Olfaction, particularly if operational in utero, may be important for identifying individuals early in life by supplementing newly developed senses. Furthermore, odor can function throughout life to enhance other visual, auditory, and tactile cues. Our sense of smell may have more impact on our life than we realize.

Acknowledgments We thank Zoto's International, Inc., for their donation of soap, shampoo, and conditioner; Graphic Connections of Geneva for their assistance in obtaining T-shirts; Dr. Jan Nyrop and the Cornell University Office of Statistical Consulting for assistance with statistical calculations; Dr. Charles E. Linn, Jr., Dr. Wendell Roelofs, Dr. Robert Johnston, Prof. Elaine Wethington, and Sarah Demo for comments on the manuscript; and the Cornell Science Inquiry Partnerships, Geneva High School, the NYS Agricultural Experiment Station, and Dr. Wendell Roelofs' laboratory for their support and assistance. This research is supported by the National Science Foundation Graduate Teaching Fellows in K-12 Education Program and Cornell University through the Cornell Science Inquiry Partnerships Program.

This research was fully researched, planned, and implemented by the following students: Maria Acquilera, Steve Alaimo, Nadine Amsel, Jenn Anderson, Chris Armstrong, Holly Baker, Mallory Barnes, Katherine Beckley, Michael Bianco, Kelsey Blowers, Ariel Brown, Alexis Carter, Jenn Case, Christi Chacchia, Jason Chan, Amy Chen, Nan Choi, Mike Crisanti, Josh Emerson, Antoine Evans, Ron Eveland, Laura Fratangelo, Kyle Frank, Rob Freeman, Morgan Fryer, Lori Guarneri, David Hall, Henry Heaton, Will Hunt, Chris Iannapollo, Hope James, Erika Kerr, Cassie Legg, Lauren Luzzi, Shanna Marshall, Samonn McCoy, Annette Michaels, Mike Moses, Elyse Nepa, Kim Notebaert, Jeannine Ocasio, Alicia Pearson, Caitlin Pratt, Kyle Shane, Krystle Smallwood, Emily Smith, Meg Spruill, Heather Stewart, Catherine Watts, Jasmin Williams, Nick Wisnieski, Jason Wirth, and Tara Wyszkowski.

\section{References}

CERnOCH, J. M. and PORTER, R. H. 1985. Recognition of maternal axillary odors by infants. Child Dev. 56: $1593-1598$.

COOLEY, M. 2001. The Columbia world of quotations, <http://www.bartleby.com>.

Devos, M., Patte, F., Rouault, J., Laffort, P., and Van Gemert, L. J. (eds.). 1990. Standardized Human Olfactory Thresholds. IRL Press, Oxford.

DотY, R. L. 1981. Olfactory communication in humans. Chem. Senses 6:351-376. 
Espelie, K. E., Wenzel, J. W., and Chang, G. 1990. Surface lipids of social wasp Polistes metricus Say and its nest and nest pedicel and their relation to nestmate recognition. J. Chem. Ecol. 16:2229-2241.

GrifFiths, S. W. and MAGURRAN, A. E. 1999. Schooling decisions in guppies (Poecilia reticulata) are based on familiarity rather than kin recognition by phenotype matching. Behav. Ecol. Sociobiol. 45:437-443.

HAYS, W. S. T. 2003. Human pheromones: Have they been demonstrated? Behav. Ecol. Sociobiol. 54:89-97.

Haze, S., Gozu, Y., Nakamura, S., Kohno, Y., Sawano, K., Ohta, H., and Yamazaki, K. 2001. 2Nonenal newly found in human body odor tends to increase with aging. J. Invest. Dermatol. 116:520-524.

HoLD, B. and SCHLEIDT, M. 1977. The importance of human odour in non-verbal communication. $Z$. Tierpsychol. 43:225-238.

Jacob, S., McClintock, M. K., Zelano, B., and Ober, C. 2002. Paternally inherited HLA alleles are associated with women's choice of male odor. Nat. Genet. 30:175-179.

KAITZ, M. and EIDELMAN, A. I. 1992. Smell recognition of newborns by women who are not their mothers. Chem. Senses 17:225-229.

KaitZ, M., Good, A., RoKem, A. M., and Eidelman, A. I. 1987. Mothers' recognition of their newborns by olfactory cues. Dev. Psychobiol. 20:587-591.

Lord, T. and KASPRZAK, M. 1989. Identification of self through olfaction. Percept. Mot. Skill. 69:219-224.

MALLET, P. and SchaAl, B. 1998. Rating and recognition of peers' personal odors by 9-year-old children: An exploratory study. J. Gen. Psychol. 125:47-64.

Mateo, J. M. 2002. Kin-recognition abilities and nepotism as a function of sociality. Proc. R. Soc. Lond., B Biol. Sci. 269:721-727.

MATEO, J. M. and JohnStOn, R. E. 2000a. Kin recognition and the 'armpit effect': Evidence of self-referent phenotype matching. Proc. R. Soc. Lond., B Biol. Sci. 267:695-700.

MATEO, J. M. and Johnston, R. E. 2000b. Retention of social recognition after hibernation in Belding's ground squirrels. Anim. Behav. 59:491-499.

Olsen, K. H., Grahn, M., Lohm, J., and LANGEFors, A. 1998. MHC and kin discrimination in juvenile Arctic charr, Salvelinus alpinus (L.). Anim. Behav. 56:319-327.

PENN, D. J. 2002. The scent of genetic compatibility: Sexual selection and the major histocompatibility complex. Ethology 108:1-21.

PlateK, S. M., Burch, R. L., and Gallup, G. G., JR. 2001. Sex differences in olfactory self-recognition. Physiol. Behav. 73:635-640.

PORTER, R. H. 1999. Olfaction and human kin recognition. Genetica 104:259-263.

Porter, R. H. and Moore, J. D. 1981. Human kin recognition by olfactory cues. Physiol. Behav. 27:493495.

Porter, R. H. and CERNOCH, J. M. 1983. Maternal recognition of neonates through olfactory cues. Physiol. Behav. 30:151-154.

Porter, R. H., Balogh, R. D., Cernoch, J. M., and Franchi, C. 1986. Recognition of kin through characteristic body odors. Chem. Senses 11:389-395.

Porter, R. H., Makin, J. W., Davis, L. D., and Christensen, K. M. 1992. Breast-fed infants respond to olfactory cues from their own mother and unfamiliar lactating females. Infant Behav. Dev. 15:85-93.

PRASAD, B. C. and REED, R. R. 1999. Chemosensation: Molecular mechanisms in worms and mammals. Trends Genet. 15:150-153.

Purves, D., Augustine, G., FitzPatrick, D., Katz, L., Lamantia, A.-S., McNamara, J., and Williams, S. 2001. The chemical senses. in (eds.) Neuroscience, 2nd ed., pp. 317-344. Sinauer Assoc., Sunderland.

Rajakaruna, R. S., Brown, J. A., Kaukinen, K., and Miller, K. M. 2001. MHC genes influence the kin recognition in juvenile Atlantic salmon. Am. Zool. 41:1562.

RusSeLL, M. J. 1976. Human olfactory communication. Nature 260:520-522.

Russell, M. J., Mendelson, T., and PeEKe, H. V. S. 1983. Mothers' identification of their infant's odors. Ethol. Sociobiol. 4:29-31.

SCHLEIDT, M. 1980. Personal odor and non-verbal communication. Ethol. Sociobiol. 1:225-231.

SChleidT, M., Hold, B., and ATTILI, G. 1981. A cross-cultural study on the attitude towards personal odors. J. Chem. Ecol. 7:19-31.

Verron, H. and Gaultier, C. 1976. Processus olfactifs et structures relationnelles. Psychol. Fr. 21:205209.

Wallace, P. 1977. Individual discrimination of humans by odor. Physiol. Behav. 19:577-579.

Weisfeld, G. E., Czilli, T., Phillips, K. A., Gall, J. A., and Lichtman, C. M. 2003. Possible olfactionbased mechanisms in human kin recognition and inbreeding avoidance. J. Exp. Child Psychol. 85:279295.

Wysocki, C. J. and Preti, G. 2000. Human body odors and chemical perception. Jpn. J. Taste Smell Res. $7: 19-42$.

Wysocki, C. J. and PretI, G. 2004. Facts, fallacies, fears and frustrations with human pheromones. Anat. Rec. 281A:1201-1211. 